\title{
Utility of scanning electron microscopy backscatter imaging for serial-sections reconstruction and postembedding immunogold detection of vesicular glutamate transporter 1 (VGLUT1) in the presynaptic terminals of the cingulate cortex
}

\author{
Cheryl Clarkson-Paredes ${ }^{1}$, Christine Brantner ${ }^{1}$ and Anastas Popratiloff ${ }^{2}$
}

${ }^{1}$ George Washington University, Nanofabricatin and Imaging Center, Washington, District of Columbia, United States, ${ }^{2}$ George Washington University, Washington, District of Columbia, United States

Postembedding immunogold (IG) labeling is a widely used method, typically employed with transmission electron microscopy (TEM) imaging. The approach enables the detection of proteins at their precise subcellular location. More importantly, a relative quantification at tens of nanometers subcellular domains is possible. The inherent value of biological electron microscopy (EM) is that the images provide comprehensive structural information following simple contrasting methods. Altogether, when the visualization of a full spectrum of subcellular structures is combined with quantitative protein analysis, the approach unfolds opportunities to engage the technique into an omics approach, such as synaptomics.

Postembedding IG limits the antigen detection to the surface of the sample, which typically requires the acquisition of serial sections for comprehensive 3-dimensional (3D) reconstruction of the objects under study. Other limitations derive from traditional EM tissue processing and the TEM imaging that have slowed down the utility of IG for neuroscience research. Antigenicity is poorly preserved in tissues processed using traditional room temperature embedding, which also alters the native structures (tissue shrinkage). To surmount this problem, a rapid freezing followed by freeze substitution emerged as an alternative to the drastic osmium fixation and resulted in dramatic improvement of protein detection. This approach has been successfully utilized in TEM. For such studies, however, TEM imaging imposes the second challenge, which is the limitation of the sample lateral dimensions which prevent large content data collection and therefore the global tissue contexts.

To overcome the above limitations, we combined backscatter scanning electron microscopy imaging with solid-state detectors on serial ultrathin sections absorbed on silicon wafers, which were previously processed by rapid freezing and freeze substitution.

In Neuroscience, postembedding immunogold labeling plays a pivotal role in understanding essential concepts in the synaptic transmission as in the interaction between the two main neuronal compartments: presynaptic and postsynaptic. Numerous studies focused on 2-dimentional (2D) expression/location of postsynaptic receptors, neuron-specific proteins associated with synaptic vesicles, and proteins involved in synaptic release. Those studies support the notion of activity-regulated roles of these molecules depending on their subdomain concentration, trafficking and synaptic location (extra synaptic, synaptic). While 2D data supported the conceptual framework at ultrastructural level, the lack of a more comprehensive 3D spatial modeling of these molecules' distribution and structural association makes it difficult to extrapolate the structure/function correlation. We tested our approach by utilizing a combination of freeze substitution/postembedding immunogold labeling of adult murine cingulate cortex sections with VGLUT1 antibody, and serial sections 3D-reconstruction. VGLUT1 is specific transporter that loads the presynaptic vesicles with glutamate in (Fig. 1).

We analyzed serial ultrathin sections from cingulate cortex embedded in Lowicryl and absorbed them on silicon wafers (Fig. 1/left panel). Serial sections were subsequently processed for a conventional postembedding IG process. Using a FEI Helios NanoLab 660 (field emission SEM) and MAPS 3.15 software (ThermoFisher), we acquired a montage of low magnifications (x1000) images, allowing the navigation and localization of a specific cortical layer (II) where there is abundance of glutamatergic terminals. Then, a corresponding selected area was captured at x35,000 relative magnification (pixel size $4.04 \mathrm{~nm}$ ) by fussing $2 \times 2$ single tile with $10 \%$ overlap (Fig. 1, middle panel). For the high-resolution imaging, we used an insertable concentric backscatter detector (CBS) in immersion mode. High-resolution imaging was sufficient to reliably identify the selected area inside of the cortical 
layer II based on characteristic cytoarchitectural structures. The region of interest included abundant neuropil and distinct labeling with VGLUT1 but also non-labeled dendrites, characteristic myelin bundles, and blood vessels that provided natural fiducial marks to identify the same region on the adjacent sections. In the next step, the same ROI was identified, and imaging was performed in the adjacent sections. In addition, a high-resolution single tile (x65,000, pixel size $2 \mathrm{~nm}$ ) was acquired for each selected pre/postsynaptic neuronal terminal labeled with VGLUT1 (Fig. 1, right panel) for 3D-reconstruction that includes the spatial distribution of the $10 \mathrm{~nm}$ gold particles, postsynaptic density, and pre and postsynaptic structures. Such reconstructions will offer comprehensive model to understand how the glutamate transporter distributes in the presynaptic terminals, how it interacts with the synaptic vesicles, and to visualize whether VGLUT1 distribution is more abundant in the vesicles withing the limits of the active zone (50 $\mathrm{nm}$ up to presynaptic membrane facing the PSD) where the vesicles are being conditioned to fuse with the presynaptic membrane.

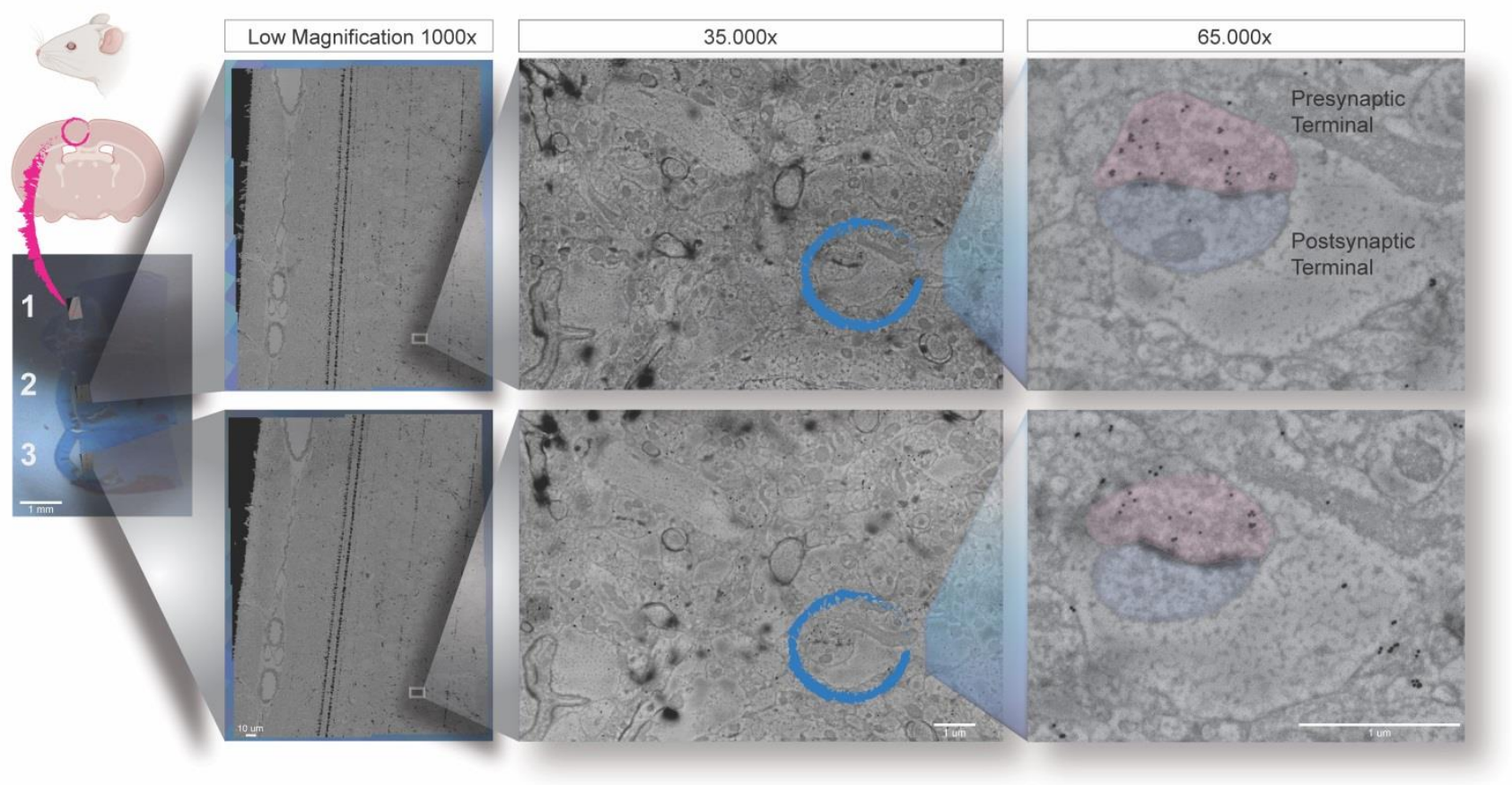

Figure 1. Illustration of the methodological approach used to identify and reconstruct the VGLUT1-labeled presynaptic terminals. Left panel, coronal section representing the location in the brain of the cingulate cortex trimmed for freeze plunge/freeze substitution. Example of a silicon wafer containing three serial sections of the cingulate cortex after postembedding immunogold process. In the second panel, representative low-mag and lowres serial SEM mapping showing the location of the middle line and the layer I and II from the cingulate cortex. Middle panel shows tile-stitching examples of two serial sections where ROI is identified. Right panel shows high magnification/high-resolution examples of a labeled presynaptic terminal and its corresponding postsynaptic structure and PSDs.

\section{References}

Clarkson C, Antunes FM, Rubio ME. Conductive Hearing Loss Has Long-Lasting

Structural and Molecular Effects on Presynaptic and Postsynaptic Structures of

Auditory Nerve Synapses in the Cochlear Nucleus. J Neurosci. 2016 Sep

28;36(39):10214-27. 